\title{
SYNCHROTRON AND SYNCHROTRON SELF-COMPTON SPECTRAL SIGNATURES AND BLAZAR EMISSION MODELS
}

\author{
JAMES ChIANG $^{1,2}$ AND MARKuS BÖTTCHER ${ }^{3.4}$ \\ Received 2001 July 31 ; accepted 2001 September 11
}

\begin{abstract}
We find that energy losses due to synchrotron self-Compton (SSC) emission in blazar jets can produce distinctive signatures in the time-averaged synchrotron and SSC spectra of these objects. For a fairly broad range of particle injection distributions, SSC-loss-dominated synchrotron emission exhibits a spectral dependence $F_{y} \sim y^{-3 / 2}$. The presence or absence of this dependence in the optical and ultraviolet spectra of flat-spectrum radio quasars such as 3C 279 and in the soft X-ray spectra of high-frequency BL Lac objects such as Mrk 501 gives a robust measure of the importance of SSC losses. Furthermore, for partially cooled particle distributions, spectral breaks of varying sizes can appear in the synchrotron and SSC spectra and will be related to the spectral indices of the emission below the break. These spectral signatures place constraints on the size scale and the nonthermal particle content of the emitting plasma, as well as the observer orientation relative to the jet axis.
\end{abstract}

Subject headings: galaxies: active - gamma rays: theory — radiation mechanisms: nonthermal

\section{INTRODUCTION}

A central uncertainty in the modeling of high-energy radiation from blazar jets is the identity of the primary energy-loss mechanism that controls the energy balance of the nonthermal plasma believed to produce the observed radiation (e.g., Sikora et al. 1997). Because of ambiguities in observer orientation relative to the jet axis and differing Doppler-beaming patterns for the various radiation processes, direct measurement and simple modeling of the high-energy spectral components cannot provide conclusive results. In this paper, we describe spectral signatures that may appear in the time-averaged synchrotron and synchrotron self-Compton (SSC) spectra of these objects. If they are present, then they provide strong evidence for the dominance of energy losses due to SSC emission rather than other processes, such as external Compton scattering or synchrotron emission.

\section{SSC COOLING AND TIME-AVERAGED SPECTRA}

We consider the cooling of nonthermal electrons in a blob of plasma that has bulk Lorentz factor $\Gamma$. Following the blazar model of Böttcher, Mause, \& Schlickeiser (1997), we assume that this blob starts off at a height $z_{i}$ above the accretion disk and travels outward along the jet axis. The nonthermal electrons and/or positrons that produce the observed synchrotron and inverse Compton (IC) emission are assumed to be injected at $z_{i}$ with an energy distribution

$$
\frac{d n}{d \gamma_{i}}=n_{0} \gamma_{i}^{-p}, \quad \gamma_{1, i} \leq \gamma_{i} \leq \gamma_{2, i},
$$

where $\gamma_{i}$ is the Lorentz factor of an individual particle, $\gamma_{2, i}$ and $\gamma_{1, i}$ are the maximum and minimum values at the time of injection, respectively, and $n_{0}$ is related to the total particle density $n_{\mathrm{tot}}$ by $n_{0}=n_{\mathrm{tot}}(1-p) /\left(\gamma_{2, i}^{1-p}-\gamma_{1, i}^{1-p}\right)$.

\footnotetext{
${ }^{1}$ NASA Goddard Space Flight Center, Code 661, Greenbelt, MD 20771.

2 Joint Center for Astrophysics and Physics Department, University of Maryland, Baltimore, MD 21250.

${ }_{3}^{3}$ Department of Physics and Astronomy, MS 61, 6100 South Main, Rice University, Houston, TX 77005-1892.

4 Chandra Fellow.
}

For synchrotron and IC cooling in the Thomson limit, the single-particle energy-loss rate is given by $\dot{\gamma}=-\beta\left(\gamma^{2}\right.$ $-1)$, where $\beta=\left(4 \sigma_{\mathrm{T}} / 3 m_{e} c\right)\left(u_{B}+u_{\mathrm{rad}}\right), m_{e}$ is the electron mass, $\sigma_{\mathrm{T}}$ is the Thomson cross section, $u_{B}=B^{2} / 8 \pi$ is the magnetic field energy density, and $u_{\mathrm{rad}}$ is the ambient photon energy density in the blob frame. If $\beta$ is constant, then for $\gamma \gg 1$, the energy of an individual particle in the comoving frame of the blob evolves as

$$
\gamma=\frac{\gamma_{i}}{1+\gamma_{i} \beta t}
$$

and the particle distribution as a function of time is

$$
\frac{d n}{d \gamma}=\frac{n_{0} \gamma^{-p}}{(1-\gamma \beta t)^{2-p}}
$$

For injection indices $p \simeq 2$, the shape of the particle spectrum remains roughly constant, becoming somewhat softer with time at the highest particle energies for $p>2$ and somewhat harder for $p<2$. The maximum and minimum Lorentz factors, $\gamma_{2}$ and $\gamma_{1}$, respectively, evolve according to equation (2), so that the entire particle spectrum marches downward in energy as time passes.

To estimate the time-averaged synchrotron spectrum, we integrate the $\delta$-function approximation of Dermer \& Schlickeiser (1993; hereafter DS93) for the instantaneous synchrotron spectral emissivity:

$$
j_{\mathrm{syn}}(\epsilon, t)=\frac{c \sigma_{\mathrm{T}}}{6 \pi \epsilon_{B}} u_{B}\left(\frac{\epsilon}{\epsilon_{B}}\right)^{1 / 2} \frac{d n}{d \gamma}\left(\left(\frac{\epsilon}{\epsilon_{B}}\right)^{1 / 2}\right),
$$

where $\epsilon=h v / m_{e} c^{2}, \epsilon_{B}=B / B_{\text {crit }}$, and $B_{\text {crit }} \equiv m_{e}^{2} c^{3} / e h=$ $4.4 \times 10^{13} \mathrm{G}$. The time-averaged spectrum is then

$$
\begin{aligned}
j_{\text {syn }}(\epsilon) & =\frac{1}{t} \int_{0}^{t} j_{s y n}(\epsilon, \tilde{t}) d \tilde{t} \\
& \simeq j_{s y n}(\epsilon, 0) \min \left(1, t_{\max } / t\right),
\end{aligned}
$$

where

$$
t_{\max }(\epsilon)=\frac{1}{\beta}\left[\left(\frac{\epsilon_{B}}{\epsilon}\right)^{1 / 2}-\frac{1}{\gamma_{2, i}}\right]
$$


is the time during which the highest energy particles emit synchrotron radiation with characteristic energy $\epsilon_{\max }=$ $\epsilon_{B} \gamma_{2}^{2}(t)>\epsilon$. The approximate relation given by equation (6) arises because of the near-constant shape of the particle distribution for $p \sim 2$. Combining equations (6) and (7), we recover the standard result for synchrotron emission from a partially cooled particle distribution:

$$
\begin{aligned}
& j_{\text {syn }}(\epsilon) \sim \epsilon^{(1-p) / 2} \quad\left(\epsilon<\epsilon_{\max }\right), \\
& j_{\text {syn }}(\epsilon) \sim \epsilon^{(1-p) / 2-1 / 2} \sim \epsilon^{-p / 2} \quad\left(\epsilon_{\max }<\epsilon\right) .
\end{aligned}
$$

This result agrees with the treatment of Dermer, Sturner, \& Schlickeiser (1997, their eq. [55]), who considered the case of synchrotron losses and losses due to Thomson scattering of external photons. Both the present derivation of equation (9) and that of Dermer et al. (1997) formally rely on $\beta$ being constant. It will still apply if $\beta$ changes on timescales much longer than the typical energy-loss timescale. For conical expansion of the jet outflow, magnetic flux freezing implies $B(z)=B_{0}\left(z / z_{i}\right)^{-\delta}$, where the index $\delta$ is of order unity. If synchrotron emission is the primary loss mechanism, then equation (9) will hold if

$$
z_{i} \gg \frac{6 \pi m_{e} c^{2} \delta \Gamma}{\sigma_{\mathrm{T}} \gamma B_{0}^{2}} \sim\left(10^{16} \mathrm{~cm}\right)\left(\frac{10^{5}}{\gamma}\right)\left(\frac{\Gamma}{10}\right)\left(\frac{1 \mathrm{G}}{B_{0}}\right)^{2}
$$

(cf. DS93). Since limits on X-ray emission due to bulk Comptonization imply injection heights of $z_{i} \gg 200 r_{g}$, where $r_{g} \equiv G M / c^{2} \sim 10^{13} \mathrm{~cm}$ for a $10^{8} M_{\odot}$ black hole (Sikora et al. 1997), we can assume the magnetic field to be effectively constant.

Energy losses due to first-order SSC emission can be estimated by using the synchrotron photon energy density:

$$
\begin{aligned}
u_{\mathrm{syn}} & \simeq \frac{4}{3} c \sigma_{\mathrm{T}} u_{B}\left(\frac{3 R_{b}}{4 c}\right) \int_{\gamma_{1}}^{\gamma_{2}} \frac{d n}{d \gamma} \gamma^{2} d \gamma \\
& \simeq \sigma_{\mathrm{T}} u_{B} R_{b} \frac{n_{0}}{3-p}\left(\gamma_{2}^{3-p}-\gamma_{1}^{3-p}\right), \quad(p \neq 3) .
\end{aligned}
$$

Here $3 R_{b} / 4 c$ is the mean residence time for photons produced in a spherical blob of radius $R_{b}$. Assuming $u_{\mathrm{syn}}>u_{B}$, $\gamma_{2} \gg \gamma_{1}$, and $p \lessgtr 3$, the single-particle energy-loss rate is

$$
\dot{\gamma} \simeq-\frac{4}{3} \frac{\sigma_{T}^{2}}{m_{e} c} R_{b} u_{B} \frac{n_{0}}{3-p} \gamma_{2}^{3-p} \gamma^{2},
$$

and the maximum particle energy evolves according to

$$
\dot{\gamma}_{2} \simeq-\tilde{\beta} \gamma_{2}^{5-p},
$$

where $\tilde{\beta} \equiv\left(4 \sigma_{\mathrm{T}}^{2} / 3 m_{e} c\right) R_{\mathrm{b}} u_{B} n_{0} /(3-p)$. In analogy to equations (6) and (7), this implies

$$
t_{\max }(\epsilon)=\frac{1}{(4-p) \tilde{\beta}}\left[\left(\frac{\epsilon_{B}}{\epsilon}\right)^{(4-p) / 2}-\frac{1}{\gamma_{2, i}^{4-p}}\right],
$$

and for the time-a veraged synchrotron spectrum, we have

$$
j_{\text {syn }}(\epsilon) \sim \epsilon^{(1-p) / 2-(4-p) / 2} \sim \epsilon^{-3 / 2} \quad\left(\epsilon_{\max }<\epsilon\right) .
$$

Here again, $\epsilon_{\max }=\epsilon_{B} \gamma_{2}^{2}$, but in this case, $\gamma_{2}$ evolves according to equation (14). Notably, the $p$-dependence drops out of the time-averaged spectrum for the highest energy synchrotron photons.

The time-averaged SSC spectrum can be estimated in a similar fashion. For this purpose, we use a similar $\delta$ - function approximation for the SSC emissivity (DS93):

$$
\begin{aligned}
j_{\mathrm{ssc}}(\epsilon, t) \simeq & \frac{c \sigma_{\mathrm{T}}^{2} u_{B} R_{b}}{9 \pi \epsilon_{B}^{1 / 2}}\left(\frac{\epsilon}{\epsilon_{B}}\right) \int_{\epsilon_{B} \gamma^{2}}^{\min \left(\epsilon_{B} \gamma_{2}^{2}, \epsilon, 1 / \epsilon\right)} d \tilde{\epsilon} \tilde{\epsilon}^{-1} \\
& \times \frac{d n}{d y}\left(\left(\frac{\tilde{\epsilon}}{\epsilon_{B}}\right)^{1 / 2}\right) \frac{d n}{d \gamma}\left(\left(\frac{\epsilon}{\tilde{\epsilon}}\right)^{1 / 2}\right) .
\end{aligned}
$$

This expression shows that the instantaneous SSC spectrum has the same spectral index, $\alpha=(p-1) / 2$ (where $F_{v} \sim v^{-\alpha}$ ), as the synchrotron component (eq. [4]). However, the maximum photon energy is instead related to the maximum electron Lorentz factor by $\epsilon_{\max }=\min \left(\gamma_{2}, \epsilon_{B} \gamma_{2}^{4}\right)$, where the former value holds for early times, but only very briefly for typical blazar parameters (see Fig. 1). Modifying equation (15) accordingly, this implies for the high-energy portion of the time-averaged SSC spectrum that

$$
j_{s s c}(\epsilon) \sim \epsilon^{(1-p) / 2-(4-p) / 4} \sim \epsilon^{-(2+p) / 4} .
$$

Even though the instantaneous synchrotron and SSC spectra have similar spectral indices, the time-averaged index of the SSC emission is harder than that of the synchrotron emission for $p \lessgtr 3$. As a corollary to this, if synchrotron emission is the most important cooling process, the time-averaged SSC spectrum scales as $j_{\mathrm{ssc}} \sim$ $\epsilon^{(1-p) / 2-1 / 4} \sim \epsilon^{(1-2 p) / 4}$ (cf. eq. [9]).

The preceding discussion assumes that the shape of the particle distribution retains the same power-law index $p$ as it evolves. Since the energy-loss rates for all the electrons (except the highest energy ones) still have a $\gamma^{2}$-dependence (eq. [13]), this continues to be approximately true for injection indices $p \sim 2$. Therefore, if SSC losses dominate the particle cooling, the synchrotron emission above the break

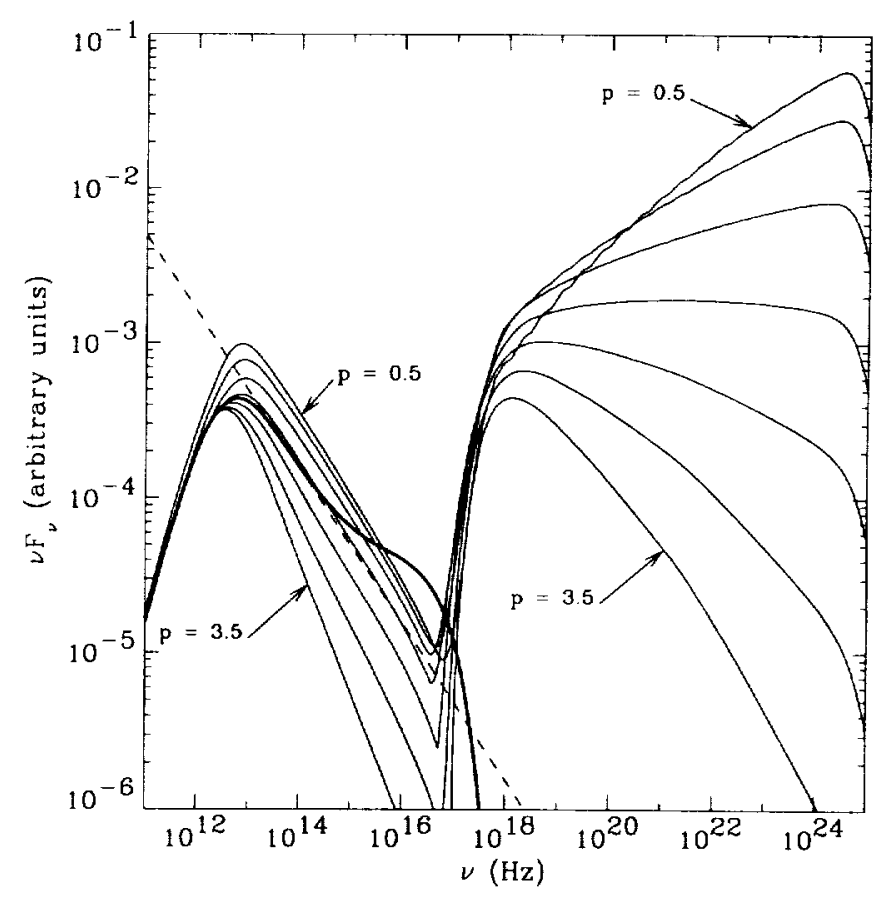

FIG. 1.-Time-averaged synchrotron and SSC spectra for values of the particle injection index $p=0.5,1,1.5,2,2.5,3 ., 3.5$ (thin curves). These spectra have been computed for parameters similar to those used for modeling the multiwaveband data of $3 \mathrm{C} 279$ for epoch P5b (Hartman et al. 2001): $\gamma_{1}=400, \gamma_{2}=10^{5}, B=1.5 \mathrm{G}, R_{b}=6 \times 10^{16} \mathrm{~cm}, n_{e}=100 \mathrm{~cm}^{-3}$. The thick curve is the time-averaged synchrotron spectrum for $p=2$, but using the full Klein-Nishina cross section to compute the SSC energy-loss rates (Böttcher et al. 1997). The dashed line shows a $F_{v} \propto v^{-3 / 2}$ spectrum. 
energy $\epsilon_{\max }$ will have a time-averaged spectral shape of $F_{v} \sim v^{-3 / 2}$ and will be relatively insensitive to the shape of the injected particle distribution. As we noted above, for $p>2$ the particle distribution becomes softer with time, while for $p<2$ it becomes harder. Thus, we would expect to see a distribution of spectral indices in the optical/UV clustered around $\alpha=3 / 2$ as the injection index varies. In the Thomson limit for SSC losses, equation (16) is a surprisingly good approximation over a broad range of injection indices. In Figure 1, we plot the time-averaged $v F_{y}$ synchrotron and SSC spectra for injection indices $p=0.5-3.5$. These spectra have been computed by numerically integrating the full expression for particle energy-loss rates, including synchrotron and SSC processes. Here we have used the formulae of Crusius \& Schlickeiser $(1986,1988)$ to compute the instantaneous synchrotron spectra, since they give more accurate results than equation (4). For $p=3$, which is at the limit of validity for equation (13), the time-averaged synchrotron spectral index is $\alpha \simeq 1.6$. Even for $p=3.5$, we find $\alpha \simeq 1.8$. If one takes the Klein-Nishina roll over into account, then the SSC energy-loss rate will deviate from the Thomson-limit expression for the highest energy electrons (Böttcher et al. 1997). This causes a flattening of the time-averaged synchrotron spectrum at the highest photon energies. The thick curve in Figure 1 is the time-averaged synchrotron spectrum for a $p=2$ calculation that uses the full Klein-Nishina cross section in determining the SSC energy-loss rates.

Another important implication of this analysis is that the spectral breaks associated with incomplete particle cooling can be significantly different from the generally assumed $\Delta \alpha=0.5$. Even for synchrotron-dominated cooling, the SSC spectral break is $\Delta \alpha=\frac{1}{4}$. If SSC cooling dominates, then a range of spectral breaks can occur. Their values will be related to the particle injection indices, and consequently to the spectral indices of the lower energy emission. For synchrotron emission, the spectral break is $\Delta \alpha=(4$ $-p) / 2=\left(3-2 \alpha_{1}\right) / 2$, where $\alpha_{1}$ is the index of the low-energy (uncooled) part of the spectrum, while for SSC emission, $\Delta \alpha=(4-p) / 4=\left(3-2 \alpha_{1}\right) / 4$. However, because of the short energy-loss timescales associated with SSC cooling for typical blazar parameters and light travel time effects through the plasma blob, detection of these spectral breaks may be difficult (Chiaberge \& Ghisellini 1999; see also $\S 4$ ). We note that the spectral breaks we discuss here are distinct from those that arise from energy cutoffs in the electron distribution function. Dermer et al. (1997) found that such breaks will be seen if they are due to a low-energy cutoff in the electron distribution or if the observation times are sufficiently short for high-energy cutoffs. For example, the lowest energy breaks in both the synchrotron and SSC components shown in Figure 1 reflect our choice of $\gamma_{1}=$ 400.

\section{APPLICATION TO BLAZAR OBSERVATIONS}

As we have shown, even if the injected electron distribution varies by a significant amount, dominant SSC cooling will produce synchrotron emission with spectral index values close to $\alpha=3 / 2$. This is precisely what has been observed for the $\gamma$-ray blazar 3C 279. Hartman et al. (2001) have used the model of Böttcher et al. (1997) and Böttcher \& Bloom (2000) to fit the infrared through $\gamma$-ray spectral energy distribution (SED) of 3C 279 for several epochs during which simultaneous multiwavelength data were taken. The Böttcher et al. model calculates, in detail, the spectra and energy losses due to synchrotron, SSC, and IC scattering of accretion-disk radiation, both directly from the disk and rescattered in the putative broad-line region (BLR). The SED fits of 3C 279, which describe the optical/ UV spectra fairly well, yield spectral indices for the synchrotron emission in these wave bands of $\alpha \simeq 1.4-1.7$ for injection indices of $p=1.9-3.1$. If synchrotron cooling were the most important process, then we would find a different range of indices, $\alpha=p / 2=0.95-1.55$.

Although the optical/UV spectral shape for $3 \mathrm{C} 279$ indicates that SSC emission is the principal cooling mechanism, this does not require the observed emission in the $\gamma$-ray band to be dominated by SSC flux. During the period in which 3 C 279 was seen to have its largest observed $\gamma$-ray flare (1996 January 30-February 6, epoch P5b of Hartman et al. 2001), the observed $\gamma$-ray flux was found, using the Böttcher et al. model, to be consistent with being mostly composed of disk and BLR IC emission, not SSC emission. However, the greater relative strength of the disk and BLR IC components is due to the fact that the assumed observer angle $\theta_{\mathrm{obs}}=2^{\circ}$ lies well within the Doppler beaming angle of $\theta_{\mathrm{D}} \simeq 1 / \Gamma=1 / 13=4^{\circ}$.4 for their calculations. As Dermer (1995) pointed out, external Compton scattered emission has a substantially narrower beaming pattern than SSC emission, and therefore has a relative magnitude greater by at least a Doppler factor ( $D \sim 20$ in this case), as compared to SSC emission for the same total luminosity. For the calculations performed for epoch P5b, the SSC component dominates the disk and BLR IC components for observing angles $\theta_{\text {obs }} \gtrsim \theta_{\mathrm{D}}$. Consistent with this, the energy-loss rates calculated by the Böttcher et al. code do show that SSC emission is the principal cooling mechanism. Application of this model to multiwavelength observations of the $\gamma$-ray blazar PKS $0528+134$ had much less success in reproducing the optical spectral shape (Mukherjee et al. 1999). The observed optical emission was significantly harder than that of 3 C 279, while the model parameters used to fit the PKS 0528+134 SEDs were consistent with cooling predominantly by SSC emission. This suggests that some other cooling mechanism controls the particle evolution, or, as was proposed by Mukherjee et al., that some additional processes, such as particle reacceleration downstream in the bulk outflow, are occurring.

BL Lac objects may provide a better "laboratory" in which to test the importance of SSC cooling than the flatspectrum radio quasars (FSRQs), as they generally do not show accretion disk components or strong broad emission lines, and the high-energy emission can often be accounted for solely by SSC emission. Since the synchrotron and SSC components have the same Doppler beaming patterns, comparing the observed luminosities of these two components indicates whether synchrotron or SSC cooling is more important. Ironically, BL Lacertae itself does occasionally exhibit weak broad UV emission lines, and Madejski et al. (1999) and Böttcher \& Bloom (2000) have shown that a BLR IC component seems to be required to explain the $\gamma$-ray emission. As with PKS $0528+134$, its optical spectrum is not well modeled by synchrotron emission from an SSC-cooled particle distribution.

This spectral diagnostic for SSC cooling need not be restricted to the optical/UV bands. For high-frequency BL Lac objects (HBLs), the synchrotron component peaks in the UV rather than in the infrared, as it does for lowfrequency BL Lac objects (LBLs), such as BL Lacertae 
(Ghisellini et al. 1998). In HBLs, the $\alpha \simeq 3 / 2$ signature of SSC cooling appears in the $X$-ray range, rather than in the optical/UV. During the 1994 May X-ray/TeV flare of the HBL Mrk 421, the X-ray energy spectral index was seen to evolve from $\alpha=1.3$ during the rise of the flare to a maximum value of $\alpha=1.5$ as the flux diminished (Takahashi et al. 1996). This spectral evolution was attributed to synchrotron cooling, but the extremal value of $\alpha=1.5$ suggests that SSC cooling may be the primary mechanism. Nearly simultaneous $\gamma$-ray fluxes measured at $100 \mathrm{MeV}$ through $\mathrm{TeV}$ energies seem to indicate that the SSC luminosity was indeed greater than the synchrotron luminosity during this flare episode (Macomb et al. 1995). By contrast, the HBL Mrk 501 does not seem to be predominantly cooled by SSC emission. The SEDs shown in Petry et al. (2000) and Katarzyński, Sol, \& Kus (2001) have a substantially harder synchrotron spectral index than $\alpha=3 / 2$, and the synchrotron luminosity consistently exceeds the SSC luminosity. The HBL PKS 2155-304 exhibited soft X-ray spectral indices of $\alpha=1.3-1.7$ during multiwavelength observations in 1994 May (Urry et al. 1997). This suggests that SSC cooling was dominant during these epochs. Unfortunately, simultaneous $\gamma$-ray coverage was not available, so direct comparisons of the SSC and synchrotron luminosities cannot be made.

\section{DISCUSSION}

If SSC emission is the primary energy-loss mechanism, then in the comoving frame of the plasma blob, the synchrotron photon energy density must exceed the energy densities associated with the other relevant loss mechanisms, synchrotron emission and IC scattering of external photons. For SSC cooling to exceed synchrotron cooling, this requires

$$
\sigma_{\mathrm{T}} R_{b} n_{\mathrm{tot}} \gamma_{2}^{3-p} \gamma_{1}^{p^{-1}}\left(\frac{p-1}{3-p}\right)>1,
$$

which follows from equation (12). This relation can yield additional constraints on the size scale and particle content of the emitting plasma by combining it with upper limits on the blob radius $R_{b}$, given by variability timescales, inferred values of the particle energy lower cutoff $\gamma_{1}$ from X-ray spectral shapes, and values of the nonthermal particle density $n_{\text {tot }}$ and magnetic field $B$ from fits to the observed optical and X-ray spectra.

Comparing the synchrotron energy density with the energy densities of external radiation is problematic because of uncertainties regarding the amount of reprocessing of the central-disk radiation that the ambient matter in the quasar environment can provide. If the synchrotron spectrum does show the signature of SSC cooling, then constraints on the properties of this ambient matter follow. Assuming it can be modeled by a spherical distribution with characteristic radius $r_{\mathrm{BLR}}$, and if the emitting plasma lies within this radius, then the energy density of reprocessed disk radiation in the blob frame is approximately ${ }^{5}$

\footnotetext{
5 The location of the emitting plasma does not appear in equation (20), since the energy density of reprocessed radiation inside a spherically symmetric shell of material that reprocesses the flux from a central point source and reemits it isotropically is approximately uniform throughout the interior of the shell. This follows from a similar line of reasoning that the net gravitational force inside a uniform shell of matter is zero everywhere within the shell.
}

$$
u_{\mathrm{BLR}} \simeq \Gamma^{2} \tau_{\mathrm{BLR}} \frac{L_{\mathrm{disk}}}{4 \pi c r_{\mathrm{BLR}}^{2}} .
$$

Here $L_{\text {disk }}$ is the disk luminosity and $\tau_{\text {BLR }}$ is the characteristic optical depth for reprocessing the disk radiation. Contributions to this optical depth may include Thomson scattering, resonant-line scattering, scattering by dust, and pure absorption (with subsequent reemission). Combining this with the expression for $u_{\text {syn }}$ (eq. [12]) and reasonable estimates of $L_{\text {disk }}$ and $\Gamma$, one can infer limits for the quantity $\tau_{\text {BLR }} / r_{\text {BLR }}^{2}$. For epoch P5b for 3C 279, Hartman et al. (2001) used $\tau_{\mathrm{BLR}}=0.003, r_{\mathrm{BLR}} \simeq 0.2 \mathrm{pc}, L_{\text {disk }}=10^{46} \mathrm{ergs} \mathrm{s}^{-1}$, and parameters similar to those used to produce Figure 1. These parameters yield $u_{\text {syn }} / u_{\mathrm{BLR}} \sim 40$, confirming the dominance of SSC cooling. A similar calculation can be applied to the direct disk radiation (DS93), and for the 3 C 279 epoch P5b model, we find $u_{\text {syn }} / u_{\text {disk }} \sim 10$.

Although we have assumed that the blazar emission is produced in a single blob of plasma, this analysis also describes the time-steady emission of a continuous jet of material in which the nonthermal electrons are injected at the base of the jet. As noted by Hartman et al. (2001), this is equivalent to a stream of discrete blobs. Even for a single blob, deviations from the time-averaged spectrum in the optical and UV wave bands may only be detectable on fairly short timescales. For the 3C 279 epoch P5b parameters, the maximum energy of the instantaneous synchrotron spectrum has swept through the optical band, after a time from the initial injection of $t_{\text {obs }}=(1+z) t_{\max } / D \sim 5$ $\times 10^{3} \mathrm{~s}$ as seen by a distant observer (see eq. [15]; here $z$ is the source redshift). In order to characterize variations in the injection of nonthermal particles or even to measure the spectral breaks associated with partially cooled particle distributions, optical and UV spectra must be taken on timescales shorter than $t_{\text {obs. }}$. However, for Mrk 421, X-ray observations during the 1994 May flare were able to detect the associated spectral evolution (Takahashi et al. 1996). This implies that the size scale and/or particle density of the emitting plasma for this object were sufficiently small to yield relatively long cooling times (see eq. [15]).

Finally, we note that this analysis underscores the role of Doppler beaming in assessing the contribution of various emission processes to the overall energy balance of the emitting plasma. Overly general assumptions about the apparent luminosity of the various inferred $\gamma$-ray components may lead one to conclude that either external IC or SSC emission is the most important process for controlling the evolution of the relativistic particles in the jet outflow, when in fact the relative strength of these components is highly dependent on observer orientation (Dermer 1995). We have shown that the presence (or absence) of a $F_{v} \sim v^{-3 / 2}$ synchrotron spectral shape provides a much more reliable measure of these processes.

We thank Bob Hartman and the anonymous referee for helpful comments that have improved this paper. M. B. is supported by NASA through Chandra Postdoctoral Fellowship Award 9-10007, issued by the Chandra X-Ray Center, which is operated by the Smithsonian Astrophysical Observatory for and on behalf of NASA, under contract NAS 8-39073. 
Böttcher, M., \& Bloom, S. D. 2000, AJ, 119, 469

\section{REFERENCES}

Böttcher M Mause H \& Schlickeiser, R. 1997, A\&A, 324, 395

Chiaberge, M., \& Ghisellini, G. 1999, MNRAS, 306, 551

Crusius, A., \& Schlickeiser, R. 1986, A\&A, 164, L16

1988, A\&A, 196, 327

Dermer, C. D. 1995, ApJ, 446, L63

Dermer, C. D., \& Schlickeiser, R. 1993, ApJ, 416, 458 (DS93)

Dermer, C. D., Sturner, S. J., \& Schlickeiser, R. 1997, ApJS, 109, 103

Ghisellini, G., et al. 1998, MNRAS, 301, 451

Hartman, R. C., et al. 2001, ApJ, 553, 683

Katarzyński, K., Sol, H., \& Kus, A. 2001, A\&A, 367, 809

Macomb, D. J., et al. 1995, ApJ, 449, L99

Madejski, G., et al. 1999, ApJ, 521, 145

Mukherjee, R., et al. 1999, ApJ, 527, 132

Petry, D., et al. 2000, ApJ, 536, 742

Sikora, M., Madejski, G., Moderski, R., \& Poutanen, J. 1997, ApJ, 484, 108

Takahashi, T., et al. 1996, ApJ, 470, L89

Urry, C. M., et al. 1997, ApJ, 486, 799 
